6. - Distance, holomorphic mappings and the Schwarz lemma, J. Math. Soc. Japan 19 (1967), 481-485.

7. - Intrinsic metrics on complex manifolds, Bull. Amer. Math. Soc. 73 (1967), 347-349.

University of CALIFornia, Berkely

\title{
A CHARACTERIZATION OF BANACH ALGEBRAS WITH APPROXIMATE UNIT
}

\author{
BY DONALD CURTIS TAYLOR
}

Communicated by R. C. Buck, February 13, 1968

1. Introduction. Let $L^{1}(R)$ denote the space of all complex valued functions on the real line $R$ which are integrable on $R$ in the sense of Lebesgue. It is well known that $L^{1}(R)$ forms a Banach algebra where the multiplication is defined by convolution; that is,

$$
f_{*} g(x)=\int_{R} f(t) g(x-t) d t
$$

and the norm of an element is defined by $\|f\|=\int|f(t)| d t$. In [4] Rudin showed that every function in $L^{1}(R)$ is the convolution of two other functions. In other words, every element of the convolution algebra $L^{1}(R)$ can be factored in $L^{1}(R)$, although this algebra lacks a unit. Subsequently, Cohen [1] observed that the essential ingredient in Rudin's argument is that $L^{1}(R)$ has an approximate unit in the sense of the following definition.

Definition. A Banach algebra $B$ is said to have an approximate unit if there exists a real number $C \geqq 1$ and a collection $\left\{e_{\lambda}: \lambda \in \Lambda\right\}$ of elements of $B$, where the index set $\Lambda$ is a directed set, such that the following two conditions are satisfied: $\left\|e_{\lambda}\right\| \leqq C$, for each $\lambda$, and $\lim e_{\lambda} x=\lim x e_{\lambda}=x$, for each $x \in B$. Cohen went on to prove that the factorization theorem still holds in any Banach algebra with approximate unit.

The results in this note stem from the observation that multiple factorization occurs in the sup-norm algebra $C_{0}(R)$, the space of all complex valued continuous functions on $R$ that vanish at infinity; that is, if $f_{1}, f_{2}, \cdots, f_{n}$ are functions in $C_{0}(R)$ and $\delta>0$, then there exist functions $g, h_{1}, h_{2}, \cdots, h_{n}$ in $C_{0}(R)$ such that

$$
f_{i}=g h_{i} \quad \text { and } \quad\left\|f_{i}-h_{i}\right\|<\delta \quad(i=1,2, \cdots, n) .
$$


Similar results are known for other Banach algebras. For example, Rudin [4, p. 111] showed that each countable collection of elements in the algebra $L^{1}(R)$ has a common divisor. Thus, the problem under consideration is the following: Does multiple factorization as given by (1.1) occur in a general setting? The answer is that in every commutative semisimple Banach algebra with approximate unit multiple factorization occurs, and furthermore, the converse is true; that is, if multiple factorization as given by (1.1) holds for a commutative semisimple Banach algebra $B$, then $B$ has an approximate unit. Our main result is the following

THEOREM 1.1. Let $B$ be a commutative semisimple Banach algebra and let $C \geqq 1$. Then the following statements are equivalent:

(1) If $Z$ is a totally bounded subset of $B$ and $\delta>0$, then there exists an element $x$ in $B,\|x\| \leqq C$, and a subset $Y$ of $B$ such that

(a) $Z=x \cdot Y$,

(b) $\|x y-y\|<\delta \quad(y \in Y)$,

(c) $Y$ is contained in the closed ideal generated by $Z$,

(d) the mapping $y \rightarrow x \cdot y$ is a homeomorphism of $Y$ onto $Z$.

(2) If $Z$ is a finite subset of $B$ and $\delta>0$, then there exists an element $x$ in $B,\|x\| \leqq C$, and a subset $Y$ of $B$ such that

(a) $Z=x \cdot Y$,

and

(b) $\|x \cdot y-y\|<\delta \quad(y \in Y)$.

(3) $B$ has an approximate unit that is uniformly bounded by $C$.

2. Notation. Let $B^{\prime}$ be the Banach algebra obtained by formally adjoining the unit $e$ to the commutative semisimple Banach algebra $B$, let $M_{B^{\prime}}$ be the maximal ideal space of $B^{\prime}$, and let $\hat{x}$ be the Gelfand transform of an element $x$ in $B^{\prime}$. (For definitions and concepts we refer the reader to [3].)

3. Proof of Theorem 1.1. We first need two preliminary results.

LEMMA 3.1. Let $B$ be a commutative semisimple Banach algebra and let $C \geqq 1$. If $B$ has an approximate unit that is uniformly bounded by $C$, then $B$ has an approximate unit $\left\{e_{\lambda}: \lambda \in \Lambda\right\}$ such that

$$
\operatorname{Re} \hat{e}_{\lambda} \geqq\left|\operatorname{Im} \hat{e}_{\lambda}\right| \text { on } M_{B^{\prime}} \text { and }\left\|e_{\lambda}\right\| \leqq C
$$

for each $\lambda$.

Proof. For a proof, see [5].

LEMMA 3.2. Let $B$ be a commutative semisimple Banach algebra and let $C \geqq 1$. Let $e_{1}, e_{2}, \cdots, e_{n+1}$ be elements in $B$ such that $\left\|e_{k}\right\| \leqq C$ and 
$\operatorname{Re} \hat{e}_{k} \geqq 0$ on $M_{B^{\prime}}$, and let $0<\lambda<1 / 4 C$. Then the elements $x_{1}, x_{2}, \cdots$, $x_{n+1}$ in $B^{\prime}$ defined by $x_{p}=\sum_{k=1}^{p} \lambda(1-\lambda)^{k-1} e_{k}+(1-\lambda)^{p} e$ are regular. Moreover, if the inequalities

$$
\left\|e_{k}-e_{n+1} e_{k}\right\|<\lambda /\left\|x_{n}^{-1}\right\|
$$

hold for $k=1,2, \cdots, n$, then there exists an element $y_{n+1}$ in $B^{\prime}$ such that

$$
x_{n+1}^{-1}=x_{n}^{-1}+\left(e-e_{n+1}\right) y_{n+1} \text { and }\left\|y_{n+1}\right\| \leqq\left\|x_{n}^{-1}\right\|^{2} \text {. }
$$

Proof. Due to the fact that $\left|\hat{x}_{p}\right|>0$ on $M_{B^{\prime}}$, we see that the elements $x_{1}, x_{2}, \cdots, x_{n+1}$ are regular. Now assume that the inequalities given by (3.2) hold. Let $g=(1-\lambda)^{n}\left[(1-\lambda) e+\lambda e_{n+1}\right]$. It is clear that $g^{-1}$ exists, $\left\|g^{-1}\right\| \leqq 2(1-\lambda)^{-n}$, and that $g^{-1}=(1-\lambda)^{-n} e+\lambda\left(e-e_{n+1}\right) g^{-1}$. Set

$$
\begin{aligned}
h & =\left[e+x_{n} g^{-1}-(1-\lambda)^{n} g^{-1}\right] \\
& =(1-\lambda)^{-n} x_{n}\left[e+(1-\lambda)^{n} x_{n}^{-1} g^{-1} \cdot \sum_{k=1}^{n} \lambda^{2}(1-\lambda)^{k-1}\left(e_{k}-e_{n+1} e_{k}\right)\right] .
\end{aligned}
$$

It follows from (3.2) that $h^{-1}$ exists and that the norm

$$
\left\|h^{-1}\right\| \leqq 2(1-\lambda)^{n}\left\|x_{n}^{-1}\right\|
$$

This fact and the identity

$$
\lambda(1-\lambda)^{n}\left(e-e_{n+1}\right) x_{n}^{-1} g^{-1}=-x_{n}^{-1}+(1-\lambda)^{n} x_{n}^{-1} g^{-1}
$$

imply that

$$
\begin{aligned}
x_{n+1}^{-1} & =\left[x_{n}+g-(1-\lambda)^{n} e\right]^{-1}=x_{n}^{-1} h^{-1} x_{n} g^{-1} \\
& =x_{n}^{-1} h^{-1}\left[h-e+(1-\lambda)^{n} g^{-1}\right] \\
& =x_{n}^{-1}+\left(e-e_{n+1}\right)\left[\lambda(1-\lambda)^{n} x_{n}^{-1} g^{-1} h^{-1}\right] .
\end{aligned}
$$

Set $y_{n+1}=\lambda(1-\lambda)^{n} x_{n}^{-1} g^{-1} h^{-1}$. It follows from (3.4) that $\left\|y_{n+1}\right\|$ $\leqq\left\|x_{n}^{-1}\right\|^{2}$ and our proof is complete.

Proof of Theorem 1.1. It is clear that (1) implies (2). We now wish to show that (2) implies (3).

Suppose (2) holds and that $\mathcal{F}$ is the family of all finite subsets of $B$. Let $\Lambda$ be the directed set defined by

$$
\Lambda=\{(Z, n): Z \in \mathcal{F}, n \text { a positive integer }\}
$$


under the usual partial ordering; that is $\left(Z_{1}, n_{1}\right) \leqq\left(Z_{2}, n_{2}\right)$ if, and only if, $Z_{1} \subset Z_{2}$ and $n_{1} \leqq n_{2}$. For each $\lambda=(Z, n) \in \Lambda$ we may choose, by virtue of (2), an element $e_{\lambda}$ in $B,\left\|e_{\lambda}\right\| \leqq C$, and a finite subset $Y_{\lambda}$ of $B$ such that

(a) $Z=e_{\lambda} \cdot Y_{\lambda}$,

(b) $\left\|e_{\lambda} y-y\right\|<1 / n\left(y \in Y_{\lambda}\right)$.

Now, for arbitrary $z \in B$ and $\epsilon>0$, choose $\lambda_{0}=(\{z\}, n)$, where $C / n<\epsilon$. Then for $\lambda \geqq \lambda_{0}$, there is a $y \in Y_{\lambda}$ such that

$$
\left\|e_{\lambda} z-z\right\|=\left\|e_{\lambda}\left(e_{\lambda} y-y\right)\right\|<C / n<\epsilon .
$$

In other words, $\lim e_{\lambda} z=z$ for every $z \in B$. Hence (2) implies (3).

To complete the proof, we need to show that (3) implies (1). Let $Z$ be a totally bounded subset of $B, 0<\lambda<\frac{1}{4} C$, and $0<\delta<1$. We shall define by induction a sequence of elements $e_{1}, e_{2}, e_{3}, \cdots$ in $B$ that is uniformly bounded by $C$ so that if

$$
x_{n}=\sum_{k=1}^{n} \lambda(1-\lambda)^{k-1} e_{k}+(1-\lambda)^{n} e,
$$

then $x_{n}^{-1}$ exists and satisfies

$$
\left\|x_{n}^{-1} z-x_{n-1}^{-1} z\right\|<\delta / 2^{n}, \quad\left\|x_{1}^{-1} z-z\right\|<\delta / 4 \quad(z \in Z) .
$$

Due to the fact $Z$ is totally bounded, we have a finite subset $F_{1}$ of $Z$ such that

$$
\sup \left\{\inf \left\{\|z-y\|: y \in F_{1}\right\}: z \in Z\right\}<\delta / 16 \text {. }
$$

Therefore, by virtue of Lemma 3.1, there exists an element $e_{1}$ in $B$, $\left\|e_{1}\right\| \leqq C$, such that $\operatorname{Re} \hat{e}_{1} \geqq 0$ on $M_{B^{\prime}}$ and that $\left\|e_{1} y-y\right\|<\delta / 4, y \in F_{1}$. It is easy to see that $x_{1}$ satisfies (3.6). Suppose that the elements $e_{1}, e_{2}, \cdots, e_{n}$ have been chosen in $B$ such that $\left\|e_{k}\right\| \leqq C$ and $\operatorname{Re} \hat{e}_{k} \geqq 0$ on $M_{B^{\prime}}$ and such that $x_{n}$ satisfies (3.6). There exists, as before, a finite subset $F_{n+1}$ of $Z$ such that

$$
\sup \left\{\inf \left\{\|z-y\|: y \in F_{n+1}\right\}: z \in Z\right\}<\lambda \cdot \delta / 2^{n+1}\left\|x_{n}^{-1}\right\|^{2}
$$

and therefore, by virtue of Lemma 3.1, there exists an element $e_{n+1}$ in $B,\left\|e_{n+1}\right\| \leqq C$, such that $\operatorname{Re} \hat{e}_{n+1} \geqq 0$ on $M_{B^{\prime}}$,

$$
\left\|e_{n+1} e_{k}-e_{k}\right\|<\lambda /\left\|x_{n}^{-1}\right\| \quad(k=1,2, \cdots, n),
$$

and 


$$
\left\|e_{n+1} y-y\right\|<\delta \cdot \lambda / 2^{n+1}\left\|x_{n}^{-1}\right\|^{2} \quad\left(y \in F_{n+1}\right) .
$$

According to Lemma 3.2, $x_{n+1}^{-1}$ exists and that

$$
x_{n+1}^{-1}-x_{n}^{-1}=\left(e-e_{n+1}\right) y_{n+1},
$$

where the norm $\left\|y_{n+1}\right\| \leqq\left\|x_{n}^{-1}\right\|^{2}$. By combining (3.7), (3.9), and (3.10) we see that (3.6) holds and therefore our induction is complete. The elements $x_{n}^{-1} z$ form a Cauchy sequence in $B$ for each element $z$ in $Z$. Therefore set

$$
Y=\left\{y=\lim x_{n}^{-1} z: z \in Z\right\} \text { and } x=\sum_{k=1}^{\infty} \lambda(1-\lambda)^{k-1} e_{k} .
$$

Since $x_{n}^{-1} z \rightarrow y$ uniformly on $Z$, we see that conditions (1a), (1c), and (1d) follow directly from the definitions of $x$ and $Y$. To see that (1b) holds, we observe that the inequalities

$$
\begin{aligned}
\|x y-y\| \leqq\left\|x y-x_{1}^{-1}(x y)\right\| & +\sum_{j=1}^{n-1}\left\|x_{j+1}^{-1}(x y)-x_{j}^{-1}(x y)\right\| \\
& +\left\|x_{n}^{-1}(x y)-y\right\|<\delta
\end{aligned}
$$

hold for each $y$ in $Y$ for the appropriate choice of $n$. Hence our proof is complete.

4. A variant of Theorem 1.1. Let $X$ be a Banach space, let $\&$ be a commutative semisimple Banach algebra of bounded operators in the Banach space $X$, and let $B X=\{T(x): T \in B, x \in X\}$. If $B$ has an approximate unit $\left\{E_{\lambda}: \lambda \in \Lambda\right\}$, then $B X=\left\{x \in X: \lim E_{\lambda}(x)=x\right\}$ and therefore $B X$ is a closed subspace of $X$ (see [2, p. 172]). We will now state a variant of Theorem 1.1 that extends a result due to P. Curtis and A. Figà-Talamanca [2, p. 171].

TheOREM 4.1. Let $X, \Theta$, and $\Theta X$ be defined as in the above paragraph and let $B$ have an approximate unit. If $Z$ is a totally bounded subset of $B X$ and $\delta>0$, then there exists an operator $T$ in $B$ and a subset $Y$ of BX such that

(1) $Z=T(Y)$,

(2) $\|T(y)-y\|<\delta \quad(y \in Y)$,

(3) $T$ is a homeomorphism of $Y$ onto $Z$.

Proof. The proof is similar to the argument given for Theorem 1.1.

Added in proof. Professor K. W. Schrader has observed that a slight modification of the proof of Lemma 3.2 will yield a result which 
allows the proof given for Theorem 1.1 to hold for an arbitrary Banach algebra and left approximate unit.

\section{REFERENCES}

1. P. Cohen, Factorization in group algebras, Duke Math. J. 26 (1959), 199-206.

2. $P$. Curtis and A. Figà-Talamanca, "Factorization theorems for Banach algebras" in Function algebras, edited by F. T. Birtel, Scott Foresman and Co., Chicago, III., 1966, pp. 169-185.

3. C. Rickart, General theory of Banach algebras, Van Nostrand, Princeton, N.J., 1960.

4. W. Rudin, Representations of functions by convolutions, J. Math. Mech. 7 (1958), 103-115.

5. D. Taylor, A generalized Fatou theorem for Banach algebras, Pacific J. Math. (to appear).

UNIVERSITY OF MISSOURI 